\title{
CASIMIR EFFECT FOR A DIELECTRIC WEDGE
}

\author{
I. Brevik and K. Pettersen \\ Division of Applied Mechanics, \\ Norwegian University of Science and Technology, \\ N-7491 Trondheim, Norway
}

November 8, 2018

\begin{abstract}
The Casimir effect is considered for a wedge with opening angle $\alpha$, with perfectly conducting walls, when the interior region is filled with an isotropic and nondispersive medium with permittivity $\epsilon$ and permeability $\mu$. The electromagnetic energy-momentum tensor in the bulk is calculated, together with the surface stress on the walls. A discussion is given on the possibilities for measuring the influence of the medium, via the Casimir-Polder force.
\end{abstract}




\section{INTRODUCTION}

The wedge geometry is an attractive system to study in connection with the Casimir effect, since the geometry is nontrivial enough to exhibit the essentials of phenomenological quantum field theory in continuous media, and yet so simple that it avoids the formal divergences that so often plague specific calculations once curved boundaries are present. An additional bonus from considering a system of this kind is that one experiences an interesting formal analogy with the theory of a straight cosmic string.

In this paper we will consider the Casimir theory of a wedge-shaped region of opening angle $\alpha$, when the walls located at angles $\theta=0$ and $\theta=\alpha$ are perfectly conducting, and the interior region $0<\theta<\alpha$ is filled with a homogeneous and isotropic dielectric medium of constant permittivity $\epsilon$ and permeability $\mu$. Figure 1 shows the geometry. The cusp is at the origin.

The present paper is a generalization of earlier work [1], in which the interior volume was taken to be a vacuum, and is also closely related to Ref. [2]. As for references to the earlier literature, we mention those listed in [1] and [2]. We may only recall here that our formalism is based upon Schwinger's source theory, as developed for the strongly related case of cylindrical geometry in [3] and [4], and is related to the general formalism given in Stratton's book [5]. A somewhat different approach is followed by Mostepanenko and Trunov in their book [6]; their Section 2.3 treats the specific wedge geometry in the vacuum case.

We ought to point out that the formulation of Casimir theory to include material properties in the bulk does not imply merely an almost trivial input of factors $\epsilon$ and $\mu$. The phenomenological electrodynamics is generally quite different from electrodynamics in a vacuum. In particular, the four-momentum of a photon in a medium as constructed on basis of Minkowski's energy-momentum tensor - or equivalently from the Hamiltonian approach - is spacelike, so it is possible to make the electrodynamic field energy negative by means of a Lorentz transformation. (Physically, it is precisely properties of this sort that are underlying the recent discussions on the so-called analog models of general relativity; cf., for instance, the papers of Leonhardt and Piwnicki [7] and the conference report in [8].)

In the following section we derive the expression for the fundamental dyad $\Gamma$, from which the effective products of the fields can be constructed. The two scalar Green functions, $F_{m}$ and $G_{m}$, are determined. In Section 4, the electromagnetic energy-momentum tensor $\Theta_{\mu \nu}$ is calculated. It is rather remarkable, as shown by Eq. (39), how the formalism conspires so as to give a very simple result: the components of $\Theta_{\mu \nu}$ reflect the presence of the medium only through a common prefactor $1 / \sqrt{\epsilon \mu}$. In Section 5 we discuss possibilities for measurements, in particular, how the deflection of an atomic beam in a medium-filled wedge is influenced by the medium. Also, the formal analogy with the theory of cosmic strings is briefly commented upon.

We put $\hbar=c=1$, and adopt electromagnetic Heaviside-Lorentz units. 


\section{DYADIC SOLUTION}

Referring to the formalism developed in [1] and [2] for the vacuum fields in the bulk, we can here be brief. We give only the basic definitions, and write down formulas when they deviate from the vacuum case. The starting point, as always when working with Schwinger's source theory, is the relationship

$$
\mathbf{E}(x)=\int d x^{\prime} \Gamma\left(x, x^{\prime}\right) \cdot \mathbf{P}(x)
$$

between the electric field $\mathbf{E}(x)$ and the polarization source $\mathbf{P}(x)$. Here $\Gamma\left(x, x^{\prime}\right)$ is the basic dyad in the formalism; its Fourier transform $\Gamma\left(\mathbf{r}, \mathbf{r}^{\prime}, \omega\right)$ follows from

$$
\Gamma\left(x, x^{\prime}\right)=\int_{-\infty}^{\infty} \frac{d \omega}{2 \pi} e^{-i \omega \tau} \Gamma\left(\mathbf{r}, \mathbf{r}^{\prime}, \omega\right),
$$

with $\tau=t-t^{\prime}$. Maxwell's equations lead to the governing equation

$$
\nabla \times \nabla \times \Gamma\left(\mathbf{r}, \mathbf{r}^{\prime}, \omega\right)-\epsilon \mu \omega^{2} \Gamma\left(\mathbf{r}, \mathbf{r}^{\prime}, \omega\right)=-\mu \omega^{2} \mathbf{1} \delta\left(\mathbf{r}-\mathbf{r}^{\prime}\right),
$$

where $\mathbf{1}$ is the unit dyad. It is advantageous to introduce a new dyad $\Gamma^{\prime}$ which is divergence-free,

$$
\Gamma^{\prime}=\Gamma+\frac{1}{\epsilon} \mathbf{1} \delta\left(\mathbf{r}-\mathbf{r}^{\prime}\right), \quad \nabla \cdot \Gamma^{\prime}=0 .
$$

The effective electric and magnetic field products are (in addition to [1-4] cf., for instance, also [9]):

$$
\begin{aligned}
i\left\langle E_{i}(\mathbf{r}) E_{j}\left(\mathbf{r}^{\prime}\right)\right\rangle_{\omega} & =\Gamma_{i j}^{\prime}\left(\mathbf{r}, \mathbf{r}^{\prime}, \omega\right) \\
i\left\langle H_{i}(\mathbf{r}) H_{j}\left(\mathbf{r}^{\prime}\right)\right\rangle_{\omega} & =-\frac{1}{\mu^{2} \omega^{2}}\left(\nabla \times \Gamma^{\prime} \times \nabla^{\prime}\right)_{i j} \\
& =\frac{1}{\mu^{2} \omega^{2}} \varepsilon_{i k l} \varepsilon_{j m n} \partial_{k} \partial_{m}^{\prime} \Gamma_{\ln }^{\prime}\left(\mathbf{r}, \mathbf{r}^{\prime}, \omega\right) .
\end{aligned}
$$

Angular brackets mean quantum mechanical expectation values. These effective products are to be inserted into the electromagnetic energy-momentum tensor $\left\langle S_{\mu \nu}\right\rangle$. The spatial components of $S_{\mu \nu}$ are

$$
S_{i k}=-E_{i} D_{k}-H_{i} B_{k}+\frac{1}{2} \delta_{i k}(\mathbf{E} \cdot \mathbf{D}+\mathbf{H} \cdot \mathbf{B}) .
$$

As $\Gamma^{\prime}$ is divergence-free, it can conveniently be expanded in cylindrical coordinates. This implies use of the vector spherical harmonics,

$$
\mathbf{X}_{l m}(\Omega)=[l(l+1)]^{-1 / 2} \mathbf{L} Y_{l m}(\Omega)
$$

As mentioned above we let $\theta$ denote the polar angle; the cusp of the wedge coincides with the $z$ axis. The boundary conditions are that the electric field is 
normal, and the magnetic field tangential, at $\theta=0, \alpha$ (se Fig.1). We let radii $\mathbf{r}$ and $\mathbf{r}^{\prime}$ correspond to $\theta, z$ and $\theta^{\prime}, z^{\prime}$ respectively, and introduce new symbols $\nu_{m}$ defined by

$$
\nu_{m}=\frac{m \pi}{\alpha},
$$

with $m$ a non-negative integer. A lengthy calculation along the lines of [1] and [2] leads to the following integral expressions for the spectral dyad $\Gamma^{\prime}$ and its double curl:

$$
\begin{aligned}
& \Gamma^{\prime}\left(\mathbf{r}, \mathbf{r}^{\prime}, \omega\right)=\frac{2}{\alpha} \sum_{m=0}^{\infty} \int_{-\infty}^{\infty} \frac{d k}{2 \pi} \\
& \times\left[-\frac{1}{\epsilon \omega^{2}}(\nabla \times \widehat{z})\left(\nabla^{\prime} \times \widehat{z}\right)\left(d_{m}-k^{2}\right) F_{m}\left(r, r^{\prime}\right) \cos \nu_{m} \theta \cos \nu_{m} \theta^{\prime}\right. \\
& \quad+\frac{1}{\epsilon \omega}(\nabla \times \nabla \times \widehat{z})\left(\nabla^{\prime} \times \nabla^{\prime} \times \widehat{z}\right) G_{m}\left(r, r^{\prime}\right) \\
& \left.\quad \times \sin \nu_{m} \theta \sin \nu_{m} \theta^{\prime}\right] e^{i k\left(z-z^{\prime}\right)}, \\
& \nabla \times \Gamma^{\prime}\left(\mathbf{r}, \mathbf{r}^{\prime}, \omega\right) \times \nabla^{\prime}=\frac{2}{\alpha} \sum_{m=0}^{\infty} \int_{-\infty}^{\infty} \frac{d k}{2 \pi} \\
& \times\left[\frac{1}{\epsilon \omega^{2}}(\nabla \times \nabla \times \widehat{z})\left(\nabla^{\prime} \times \nabla^{\prime} \times \widehat{z}\right)\left(d_{m}-k^{2}\right) F_{m}\left(r, r^{\prime}\right) \cos \nu_{m} \theta \cos \nu_{m} \theta^{\prime}\right. \\
& -\frac{1}{\epsilon \omega}(\nabla \times \widehat{z})\left(\nabla^{\prime} \times \widehat{z}\right)\left(d_{m}-k^{2}\right)\left(d_{m}^{\prime}-k^{2}\right) G_{m}\left(r, r^{\prime}\right) \\
& \left.\times \sin \nu_{m} \theta \sin \nu_{m} \theta^{\prime}\right] e^{i k\left(z-z^{\prime}\right) .}
\end{aligned}
$$

Here $k \in\langle-\infty, \infty\rangle$ is the axial wave number, the prime on the summation sign means that the $m=0$ term is taken with half weight, $\widehat{z}$ is the unit vector in the $z$ direction, and $F_{m}, G_{m}$ are the two scalar Green functions. Further, $d_{m}$ is the differential operator

$$
d_{m}=\left(\frac{1}{r} \frac{\partial}{\partial r} r \frac{\partial}{\partial r}-\frac{\nu_{m}^{2}}{r^{2}}\right) .
$$

The scalar Green functions are explicitly

$$
\begin{aligned}
F_{m}\left(r, r^{\prime}\right) & =\frac{\omega^{2}}{q^{2}}\left[\mathcal{G}_{m}^{F}\left(r, r^{\prime}\right)+\frac{i \pi}{2} J_{\nu_{m}}\left(q r_{<}\right) H_{\nu_{m}}\left(q r_{>}\right)\right], \\
G_{m}\left(r, r^{\prime}\right) & =\frac{\omega}{q^{2}}\left[\mathcal{G}_{m}^{G}\left(r, r^{\prime}\right)+\frac{i \pi}{2} J_{\nu_{m}}\left(q r_{<}\right) H_{\nu_{m}}\left(q r_{>}\right)\right],
\end{aligned}
$$

where

$$
q^{2}=\epsilon \mu \omega^{2}-k^{2},
$$


$J_{\nu_{m}}$ and $H_{\nu_{m}}$ being ordinary Bessel and Hankel functions of order $\nu_{m}$. Further, $\mathcal{G}_{m}^{F, G}$ are the so-called auxiliary Green functions, given by

$$
\begin{gathered}
\mathcal{G}_{m}^{F, G}=-\frac{1}{2 \nu_{m}}\left(\frac{r_{<}}{r_{>}}\right)^{\nu_{m}}, m>0, \\
\mathcal{G}_{0}^{F, G}=-\frac{1}{2} \ln \frac{r_{<}}{r_{>}} .
\end{gathered}
$$

It ought to be noted that the Green functions are derived on the basis of requiring boundedness as $r \rightarrow 0$, and outgoing wave conditions as $r \rightarrow \infty$.

\section{EFFECTIVE FIELD PRODUCTS}

Using Eqs. (5), (6) and (10), (11) we can now calculate the effective field product within the wedge, assuming that the two spacetime points $x$ and $x^{\prime}$ are separated. In full generality, all coordinates $\{t, r, \theta, z\}$ would be different from $\left\{t^{\prime}, r^{\prime}, \theta^{\prime}, z^{\prime}\right\}$. However, we assume henceforth $t=t^{\prime}$ and $z=z^{\prime}$, so that $x$ and $x^{\prime}$ are separated only spatially, in the radial and azimuthal directions.

It is first to be noted that the functions $\mathcal{G}_{0}^{F, G}$ do not contribute to the effective products. The argument runs similarly to that in [1]. As for the auxiliary Green functions we are left only with $\mathcal{G}_{m}^{F, G}$ with $m>0$.

Now introducing for convenience the operator

$$
\mathcal{L}=\frac{2}{\alpha} \frac{1}{(2 \pi)^{2}} \int_{-\infty}^{\infty} d \omega \int_{-\infty}^{\infty} d k \sum_{m=0}^{\infty},
$$

we obtain for the diagonal effective products

$$
\begin{aligned}
& i\left\langle E_{\theta}(r, \theta) E_{\theta}\left(r^{\prime}, \theta^{\prime}\right)\right\rangle=\mathcal{L}\left[\frac { i \pi } { 2 } \left\{\mu \omega^{2} J_{\nu_{m}}^{\prime}\left(q r_{<}\right) H_{\nu_{m}}^{\prime}\left(q r_{>}\right)\right.\right. \\
& \left.\left.+\frac{\nu_{m}^{2} k^{2}}{\epsilon q^{2}} \frac{1}{r r^{\prime}} J_{\nu_{m}}\left(q r_{<}\right) H_{\nu_{m}}\left(q r_{>}\right)\right\} \cos \nu_{m} \theta \cos \nu_{m} \theta^{\prime}\right], \\
& i\left\langle H_{\theta}(r, \theta) H_{\theta}\left(r^{\prime}, \theta^{\prime}\right)\right\rangle=\mathcal{L}\left[\frac { i \pi } { 2 } \left\{\epsilon \omega^{2} J_{\nu_{m}}^{\prime}\left(q r_{<}\right) H_{\nu_{m}}^{\prime}\left(q r_{>}\right)\right.\right. \\
& \left.\left.+\frac{\nu_{m}^{2} k^{2}}{\mu q^{2}} \frac{1}{r r^{\prime}} J_{\nu_{m}}\left(q r_{<}\right) H_{\nu_{m}}\left(q r_{>}\right)\right\} \sin \nu_{m} \theta \sin \nu_{m} \theta^{\prime}\right], \\
& i\left\langle E_{r}(r, \theta) E_{r}\left(r^{\prime}, \theta^{\prime}\right)\right\rangle=\mathcal{L}\left[\frac { i \pi } { 2 } \left\{\frac{k^{2}}{\epsilon} J_{\nu_{m}}^{\prime}\left(q r_{<}\right) H_{\nu_{m}}^{\prime}\left(q r_{>}\right)\right.\right. \\
& \left.\left.+\frac{\nu_{m}^{2} \mu \omega^{2}}{q^{2}} \frac{1}{r r^{\prime}} J_{\nu_{m}}\left(q r_{<}\right) H_{\nu_{m}}\left(q r_{>}\right)\right\} \sin \nu_{m} \theta \sin \nu_{m} \theta^{\prime}\right],
\end{aligned}
$$




$$
\begin{gathered}
i\left\langle H_{r}(r, \theta) H_{r}\left(r^{\prime}, \theta^{\prime}\right)\right\rangle=\mathcal{L}\left[\frac { i \pi } { 2 } \left\{\frac{k^{2}}{\mu} J_{\nu_{m}}^{\prime}\left(q r_{<}\right) H_{\nu_{m}}^{\prime}\left(q r_{>}\right)\right.\right. \\
\left.\left.+\frac{\nu_{m}^{2} \epsilon \omega^{2}}{q^{2}} \frac{1}{r r^{\prime}} J_{\nu_{m}}\left(q r_{<}\right) H_{\nu_{m}}\left(q r_{>}\right)\right\} \cos \nu_{m} \theta \cos \nu_{m} \theta^{\prime}\right] \\
i\left\langle E_{z}(r, \theta) E_{z}\left(r^{\prime}, \theta^{\prime}\right)\right\rangle=\mathcal{L}\left[\frac{i \pi}{2} \frac{q^{2}}{\epsilon} J_{\nu_{m}}\left(q r_{<}\right) H_{\nu_{m}}\left(q r_{>}\right) \sin \nu_{m} \theta \sin \nu_{m} \theta^{\prime}\right] \\
i\left\langle H_{z}(r, \theta) H_{z}\left(r^{\prime}, \theta^{\prime}\right)\right\rangle \\
=\mathcal{L}\left[\frac{i \pi}{2} \frac{q^{2}}{\mu} J_{\nu_{m}}\left(q r_{<}\right) H_{\nu_{m}}\left(q r_{>}\right) \cos \nu_{m} \theta \cos \nu_{m} \theta^{\prime}\right]
\end{gathered}
$$

These are the diagonal products needed to calculate the normal stresses on the surface. There are nondiagonal field products also, $\left\langle E_{i} E_{k}\right\rangle$ and $\left\langle H_{i} H_{k}\right\rangle$, with $i \neq k$. These products do not vanish by themselves, but it is notable that the sum $\epsilon\left\langle E_{i} E_{k}\right\rangle+\mu\left\langle H_{i} H_{k}\right\rangle$, present in the electromagnetic stress tensor, does vanish.

It is also to be noted that the auxiliary Green functions $\mathcal{G}_{m}^{F, G}$ with $m>0$ do not contribute to the field products. This is because of the relations $d_{m} \mathcal{G}_{m}^{F, G}=0$, as well as

$$
\begin{gathered}
\left(d_{m}+\epsilon \mu \omega^{2}-k^{2}\right) F_{m}=\omega^{2} \mathcal{G}_{m}^{F}, \\
\left(d_{m}+\epsilon \mu \omega^{2}-k^{2}\right) G_{m}=\omega \mathcal{G}_{m}^{G} .
\end{gathered}
$$

Finally, one may verify by explicit calculation that the Poynting vector vanishes. This is as one would expect, under stationary conditions.

It is to be emphasized that the differences $\left(r-r^{\prime}\right)$ and $\left(\theta-\theta^{\prime}\right)$ are at the present stage of the calculation arbitrary; they are not necessarily small.

\section{ENERGY-MOMENTUM TENSOR}

We now let the two points $r$ and $r^{\prime}$ approach each other, but keep both $\left(r-r^{\prime}\right)$ and $\left(\theta-\theta^{\prime}\right)$ different from zero. For convenience we write henceforth $\left\langle E_{r}^{2}\right\rangle$ instead of $\left\langle E_{r}(r, \theta) E_{r}\left(r^{\prime}, \theta^{\prime}\right)\right\rangle_{\mathbf{r} \rightarrow \mathbf{r}^{\prime}}$, etc. The azimuthal diagonal component of $\left\langle S_{\mu \nu}\right\rangle$ at an arbitrary position $\mathbf{r}$ within the wedge is

$$
\begin{aligned}
\left\langle S_{\theta \theta}(\mathbf{r})\right\rangle= & \frac{1}{2}\left[\epsilon\left\langle E_{r}^{2}\right\rangle-\epsilon\left\langle E_{\theta}^{2}\right\rangle+\epsilon\left\langle E_{z}^{2}\right\rangle\right. \\
& \left.+\mu\left\langle H_{r}^{2}\right\rangle-\mu\left\langle H_{\theta}^{2}\right\rangle+\mu\left\langle H_{z}^{2}\right\rangle\right] .
\end{aligned}
$$

Inserting the effective products above we get

$$
\begin{aligned}
\left\langle S_{\theta \theta}(\mathbf{r})\right\rangle= & \frac{\pi}{4} \mathcal{L}\left[\left\{q^{2}+\frac{1}{r r^{\prime}} \frac{\partial}{\partial \theta} \frac{\partial}{\partial \theta^{\prime}}-\frac{\partial}{\partial r} \frac{\partial}{\partial r^{\prime}}\right\}\right. \\
& \left.\times J_{\nu_{m}}\left(q r_{<}\right) H_{\nu_{m}}\left(q r_{>}\right) \cos \nu_{m}\left(\theta-\theta^{\prime}\right)\right]_{\mathbf{r} \rightarrow \mathbf{r}^{\prime}} .
\end{aligned}
$$


From this we have to subtract off the contact term, called $\left\langle S_{\theta \theta}^{0}(\mathbf{r})\right\rangle$. As the Casimir effect is caused by the boundaries of the wedge, it follows that the contact term has to be evaluated in the absence of any boundaries at all. In other words, $\left\langle S_{\theta \theta}^{0}(\mathbf{r})\right\rangle$ corresponds to a homogeneous dielectric extending over all space. Explicit calculation shows that the contact term becomes equal to the wedge effective product evaluated at $\alpha=\pi$ :

$$
\left\langle S_{\theta \theta}^{0}(\mathbf{r})\right\rangle=\left\langle S_{\theta \theta}^{\alpha=\pi}(\mathbf{r})\right\rangle .
$$

Altough this is a natural result, it could hardly have been written down beforehand, without explicit calculation.

We perform a complex frequency rotation, $\omega \rightarrow i \widehat{\omega}$, implying

$$
q=\sqrt{\epsilon \mu \omega^{2}-k^{2}} \rightarrow \sqrt{-\left(\epsilon \mu \widehat{\omega}^{2}+k^{2}\right)} \equiv i \rho .
$$

Then,

$$
\begin{aligned}
& \left\langle S_{\theta \theta}(\mathbf{r})\right\rangle=\frac{1}{\alpha \pi^{2}} \int_{-\infty}^{\infty} d \widehat{\omega} \int_{0}^{\infty} d k \sum_{m=0}^{\infty}{ }^{\prime}\left[\left\{-\rho^{2}+\frac{1}{r r^{\prime}} \frac{\partial}{\partial \theta} \frac{\partial}{\partial \theta^{\prime}}-\frac{\partial}{\partial r} \frac{\partial}{\partial r^{\prime}}\right\}\right. \\
& \left.\times I_{\nu_{m}}\left(\rho r_{<}\right) K_{\nu_{m}}\left(\rho r_{>}\right) \cos \nu_{m}\left(\theta-\theta^{\prime}\right)\right]_{\mathbf{r} \rightarrow \mathbf{r}^{\prime}},
\end{aligned}
$$

where $I_{\nu_{m}}$ and $K_{\nu_{m}}$ are modified Bessel functions. We go over to polar coordinates, noting that $\sqrt{\epsilon \mu} d \widehat{\omega} d k \rightarrow \rho d \rho d \phi$ since $\rho$ is the radius in the $\sqrt{\epsilon \mu} \widehat{\omega}, k$ plane. Integrating over angles $\phi$ from 0 to $\pi / 2$ we obtain

$$
\begin{aligned}
& \left\langle S_{\theta \theta}(\mathbf{r})\right\rangle= \\
& \frac{1}{2 \pi \alpha \sqrt{\epsilon \mu}} \sum_{m=0}^{\infty} \int_{0}^{\infty} \rho d \rho\left[\left\{-\rho^{2}+\frac{1}{r r^{\prime}} \frac{\partial}{\partial \theta} \frac{\partial}{\partial \theta^{\prime}}-\frac{\partial}{\partial r} \frac{\partial}{\partial r^{\prime}}\right\}\right. \\
& \left.\times I_{\nu_{m}}\left(\rho r_{<}\right) K_{\nu_{m}}\left(\rho r_{>}\right) \cos \nu_{m}\left(\theta-\theta^{\prime}\right)\right]_{\mathbf{r} \rightarrow \mathbf{r}^{\prime}} .
\end{aligned}
$$

We now introduce

$$
p=\frac{\pi}{\alpha},
$$

and assume henceforth that $p$ is an integer. Therewith $\nu_{m}=m p$ also becomes an integer. This simplifying case is convenient, as it allows us to make use of the generalized Graf addition theorem for modified Bessel functions. The important formula in our context is given by Eq. (B.6) in [1], and will not be repeated here. By means of it, we get

$$
\begin{aligned}
& \left\langle S_{\theta \theta}(\mathbf{r})\right\rangle=\frac{1}{(2 \pi)^{2} \sqrt{\epsilon \mu}} \int_{0}^{\infty} \rho d \rho \\
& \times\left.\left[-\rho^{2}+\frac{1}{r r^{\prime}} \frac{\partial}{\partial \theta} \frac{\partial}{\partial \theta^{\prime}}-\frac{\partial}{\partial r} \frac{\partial}{\partial r^{\prime}}\right] \sum_{m=0}^{p-1} K_{0}\left(\rho R_{n}\right)\right|_{\mathbf{r} \rightarrow \mathbf{r}^{\prime}},
\end{aligned}
$$


where

$$
R_{n}=\left[r^{2}+r^{\prime 2}-2 r r^{\prime} \cos \left(\left(\theta-\theta^{\prime}\right)+\frac{2 \pi n}{p}\right)\right]^{1 / 2} .
$$

This expression can be processed further, using Eqs. (56) - (60) in [1]. The regularized energy-momentum tensor $\left\langle\Theta_{\mu \nu}(\mathbf{r})\right\rangle$, as defined generally by

$$
\left\langle\Theta_{\mu \nu}(\mathbf{r})\right\rangle=\left\langle S_{\mu \nu}(\mathbf{r})\right\rangle-\left\langle S_{\mu \nu}^{0}(\mathbf{r})\right\rangle,
$$

then yields for the $\theta \theta$ component

$$
\left\langle\Theta_{\theta \theta}(\mathbf{r})\right\rangle=-\frac{3}{720 \pi^{2} \sqrt{\epsilon \mu} r^{4}}\left(\frac{\pi^{2}}{\alpha^{2}}+11\right)\left(\frac{\pi^{2}}{\alpha^{2}}-1\right) .
$$

Similar considerations can be carried out for the other components of the energymomentum tensor. If we numerate the components according to $\left\langle\Theta_{\mu \nu}\right\rangle=$ $\left\langle\Theta_{r r}, \Theta_{\theta \theta}, \Theta_{z z},-w\right\rangle$ where $w$ is the electromagnetic energy density, we get finally

$$
\left\langle\Theta_{\mu \nu}(\mathbf{r})\right\rangle=\frac{1}{720 \pi^{2} \sqrt{\epsilon \mu} r^{4}}\left(\frac{\pi^{2}}{\alpha^{2}}+11\right)\left(\frac{\pi^{2}}{\alpha^{2}}-1\right) \operatorname{diag}(1,-3,1,1) .
$$

These expressions all vanish for $\pi=\alpha$, as expected.

The simplicity of the expression (39) is rather remarkable. The expression differs from the corresponding expression in vacuum [1] only through the factor $\sqrt{\epsilon \mu}$ in the denominator. A value $n=\sqrt{\epsilon \mu}>1$ of the refractive index $n$ thus causes the energy density in the bulk, as well as the normal stress on the plates, to be less than the vacuum value. In the special case of $\epsilon \mu=1$, a case considered repeatedly in recent years in various contexts (a so-called "relativistic" medium), one ends up with precisely the same energy-momentum tensor as in vacuum. There seems to be no simple way to see beforehand why the dependence on the properties of the medium should have the special form of Eq. (39).

\section{ON POSSIBILITIES FOR EXPERIMENTS}

We consider first the normal surface density on the lower wall, $\theta=0$, as a function of the distance $r$ from the cusp. For convenience we will denote this force density by $\sigma(r)$. Since our triplet of basis vectors in the spatial directions $\{r, \theta, z\}$ is orthonormal, $\sigma(r)$ must simply be equal to $-\left\langle S_{\theta \theta}(\mathbf{r})\right\rangle$, taken at the wall. Thus, in dimensional units,

$$
\sigma(r)=\frac{\hbar c}{720 \pi^{2} \sqrt{\epsilon \mu} r^{4}}\left(\frac{\pi^{2}}{\alpha^{2}}+11\right)\left(\frac{\pi^{2}}{\alpha^{2}}-1\right) .
$$

The force between the walls is attractive, as expected, and it decreases quickly when one moves away from the cusp. The divergence at the cusp is clearly 
fictitious: the presence of a skin depth $\delta$ in the material forbids us to apply the continuum dielectric model at very small distances. Assume copper, for instance, for which the conductivity is $6.0 \times 10^{7}(\Omega \mathrm{m})^{-1}$. As the most significant frequencies contributing to the Casimir force are of the order $c / a$, where $a$ is the local separation between the walls, we obtain $\delta \sim 10 \mathrm{~nm}$ if $a \sim 1 \mu \mathrm{m}$ (the typical separation distance) [1]. The expression (40) is hardly applicable until $r$ becomes as large as about $1 \mu \mathrm{m}$.

Assume for definiteness a very narrow wedge, corresponding to $\alpha=10^{-4} \mathrm{rad}$ $\left(0.0057^{\circ}\right)$. A local wall separation of $a=1 \mu m$ then corresponds to $r=a / \alpha=$ $1 \mathrm{~cm}$. From Eq. (40) we get

$$
\sigma(r=1 \mathrm{~cm})=\frac{0.0043}{\sqrt{\epsilon \mu}} \frac{d y n}{c m^{2}}
$$

This is about $(3 \sqrt{\epsilon \mu})^{-1}$ of the conventional surface force density $0.013 \mathrm{dyn} / \mathrm{cm}^{2}$ between two parallel perfectly conducting plates at the same separation as above, $a=1 \mu \mathrm{m}$.

It does not seem to be easy to design a surface force experiment of this kind. A more promising possibility might be to consider a variant of the deflection experiment of Sukenik et al. [10]. These authors measured the deflection of a ground-state atomic beam passing through a wedge-shaped cavity of opening angle $\alpha \sim 10^{-4} \mathrm{rad}$, thus of the same order of magnitude as considered above. Let us assume the wedge region to be completely filled with a fluid, having material constants $\epsilon$ and $\mu$. The transverse force on the beam, causing the deflection, will clearly be $\epsilon$ and $\mu$ dependent. This transverse force is of course the Casimir-Polder rather than the Casimir force in the strict sense, but the two kinds of forces are strongly interrelated.

Let us calculate the interaction energy $U(\mathbf{r})$ for a dipolar particle in the wedge as if the particle were at rest, at position $\mathbf{r}$. It is natural to assume that retardation effects are not important, so that we can use the material properties of the medium at $\omega=0$ with satisfactory accuracy. Thus, we can use the static polarizability $\alpha(0)$ for the particle, and so obtain

$$
U(\mathbf{r})=-\frac{1}{2} \alpha(0)\left\langle\mathbf{E}^{2}\right\rangle=-\frac{1}{2} \alpha(0)\left[\left\langle E_{r}^{2}\right\rangle+\left\langle E_{\theta}^{2}\right\rangle+\left\langle E_{z}^{2}\right\rangle\right] .
$$

Using Eqs. (19), (21) and (23) we obtain after some calculation, putting $\theta=\theta^{\prime}$,

$$
\begin{aligned}
U(\mathbf{r})= & -\frac{\alpha(0)}{4 \pi \alpha \sqrt{\epsilon \mu}} \frac{1}{\epsilon} \int_{0}^{\infty} \rho^{3} d \rho \\
& \times \sum_{m=0}^{\infty}\left[I_{m p}^{\prime}(m p \theta) K_{m p}^{\prime}(m p \theta) \cos 2 m p \theta\right. \\
& -\frac{m^{2} p^{2}}{\rho^{2} r r^{\prime}} I_{m p}\left(\rho r_{<}\right) K_{m p}\left(\rho r_{>}\right) \cos 2 m p \theta \\
& \left.-2 I_{m p}\left(\rho r_{<}\right) K_{m p}\left(\rho r_{>}\right) \sin ^{2} m p \theta\right]
\end{aligned}
$$


Recall that $p=\pi / \alpha$ has been assumed to be an integer. On physical grounds, $r$ and $r^{\prime}$ have to lie close to each other in the expression (43). The expression may be regularized: if one subtracts off a contact term corresponding to $p=1$ $(\alpha=\pi)$, one is left with the wedge-specific contribution, equal to zero in the case of a single plane plate in interaction with the dipole.

The expression (43) has the important property that the influence from the medium turns up only in the prefactor $(\sqrt{\epsilon \mu} \epsilon)^{-1}$. That is, we can make use of the results derived earlier for the case of a vacuum wedge [2]. Defining $\xi=r_{<} / r_{>}$and taking $(\xi-1)$ to be small, we obtain using Eq. (3.10) in [2]:

$$
\begin{aligned}
U(\mathbf{r})= & -\frac{\alpha(0)}{16 \pi^{2} \sqrt{\epsilon \mu} \epsilon r^{4}} \\
& \times\left[\frac{3}{2} \frac{p^{4}}{\sin ^{4} p \theta}-\frac{p^{2}\left(p^{2}-1\right)}{\sin ^{2} p \theta}-\frac{1}{90}\left(p^{2}+11\right)\left(p^{2}-1\right)\right] .
\end{aligned}
$$

Assuming $\sqrt{\epsilon \mu} \epsilon>1$ which is usually the case, we thus find a weaker deflection of the point dipole toward the nearby wall than in the case of a vacuum cavity. In the special case of a plane plate $(p=1)$, only the first term in (44) survives.

\section{CONCLUSIONS AND FURTHER REMARKS}

Let us first summarize:

(1) We assumed nondispersive permittivity $\epsilon$ and permeability $\mu$ in the medium-filled wedge. All calculations were made at zero temperature. The electromagnetic energy-momentum tensor $\left\langle\Theta_{\mu \nu}\right\rangle$, after regularization, is given by Eq. (39). All components of $\left\langle\Theta_{\mu \nu}\right\rangle$ are independent of the polar angle $\theta$ and diminish with distance $r$ from the cusp as $r^{-4}$. The influence from the medium turns up solely in the prefactor $\sqrt{\epsilon \mu}$ in the denominator.

(2) There exists to our knowledge no experiment testing the present kind of theory. The most promising possibility seems to be the dielectric variant of the experiment of Sukenik et al. [10], measuring the deflection of an atomic beam passing through a wedge-shaped cavity of small opening angle $\alpha$. If the wedge is filled with a medium, the transverse force on a dipole is given by the gradient of the potential (44), showing that the presence of the medium turns up solely in the prefactor $\sqrt{\epsilon \mu} \epsilon$ in the denominator.

Then a couple of further remarks:

(3) The formulation of the theory at finite temperatures can be carried out in the conventional way, replacing the integral over imaginary frequencies $\widehat{\omega}$ by a sum over discrete Matsubara frequencies $\widehat{\omega}_{n}=\left(2 \pi n k_{B} T\right)$, with $n$ an integer. This procedure was shown in [1] in detail for the component $\left\langle S_{\theta \theta}(\mathbf{r})\right\rangle^{T}$, thus generalizing the expression (32) above to the case of finite temperatures, and will 
not be further considered here. Again, the important point in our context is the apperance of the extra factor $\sqrt{\epsilon \mu}$ in the denominator in the energy-momentum tensor expression.

(4) Finally, it is worth noticing that the interesting formal analogy that exists between a wedge and a straight cosmic string can be carried over to the medium case, only with a slight modification. The line element outside a string is

$$
d s^{2}=-d t^{2}+d r^{2}+(1-4 G \mu)^{2} r^{2} d \theta^{2}+d z^{2},
$$

where $G$ is the gravitational constant and $\mu \simeq 10^{22} \mathrm{~g} / \mathrm{cm}$ is the string mass per unit length (GUT scale). Defining the symbol $\beta=(1-4 G \mu)^{-1}$, we can write the electromagnetic energy-momentum tensor as [11]

$$
\left\langle\Theta_{\mu \nu}\right\rangle=\frac{1}{720 \pi^{2} r^{4}}\left(\beta^{2}+11\right)\left(\beta^{2}-1\right) \operatorname{diag}(1,-3,1,1) .
$$

Comparison with (39) shows that, apart from the extra prefactor $\sqrt{\epsilon \mu}$ in the denominator of that equation, there is complete analogy between the two cases if the gravitational quantity $\beta$ is identified with the wedge quantity $\pi / \alpha$. 


\section{REFERENCES}

[1] I. Brevik and M. Lygren, Ann. Phys. (N.Y.) 251 (1996), 157.

[2] I. Brevik, M. Lygren and V. N. Marachevsky,

Ann. Phys. (N.Y.) 267 (1998), 134.

[3] I. Brevik and G. H. Nyland,

Ann. Phys. (N.Y.) 230 (1994), 321.

[4] L. L. DeRaad, Jr. and K. A. Milton,

Ann. Phys. (N.Y.) 136 (1981), 229.

[5] J. A. Stratton,

"Electromagnetic Theory", McGraw - Hill, New York, 1941.

[6] V. M. Mostepanenko and N. N. Trunov,

"The Casimir Effect and its Applications", Clarendon Press, Oxford, 1997.

[7] U. Leonhardt and P. Piwnicki,

Phys. Rev. A 60 (1999), 4301; Phys. Rev. Lett. 84 (2000), 822.

[8] Workshop on "Analog models of General Relativity",

Rio de Janeiro, Brazil, October 2000. Address: www.lafex.cbpf.br/ ${ }^{\sim} b s c g / a n a l o g /$

[9] K. A Milton,

Ann. Phys. (N.Y.) 127 (1980), 49.

[10] C. I. Sukenik, M. G. Boshier, D. Cho, V. Sandoghdar and E. A. Hinds, Phys Rev. Lett. 70 (1993), 560.

[11] V. P. Frolov and E. M. Serebriany,

Phys. Rev. D 35 (1987), 3779. 